\title{
Laboratoire de physiologie de la perception et de l'action
}

UMR 7152 CNRS-Collège de France

\section{Sidney Wiener}

\section{OpenEdition}

\section{Journals}

Édition électronique

URL : https://journals.openedition.org/annuaire-cdf/1273

DOI : 10.4000 /annuaire-cdf. 1273

ISBN : 978-2-7226-0325-7

ISSN : 2109-9227

\section{Éditeur}

Collège de France

\section{Édition imprimée}

Date de publication : 1 avril 2013

Pagination : 871-879

ISBN : 978-2-7226-0198-7

ISSN : 0069-5580

\section{Référence électronique}

Sidney Wiener, « Laboratoire de physiologie de la perception et de l'action », L'annuaire du Collège de France [En ligne], 112 | 2013, mis en ligne le 22 novembre 2013, consulté le 22 août 2022. URL : http:// journals.openedition.org/annuaire-cdf/1273; DOI : https://doi.org/10.4000/annuaire-cdf.1273 
droit. Depuis sa $\mathrm{IV}^{\mathrm{e}}$ rencontre, à Paris, les 30 juin et $1^{\mathrm{er}}$ juillet 2011 à la Fondation Hugot, le réseau franco-brésilien a continué de travailler sur les mutations de la responsabilité en vue de sa participation à la Rencontre inter-réseaux à Paris les 10 , 11 et 12 avril 2012, respectivement à la Fondation Hugot, au Collège de France et au ministère des Affaires étrangères et européennes, dont le thème était «l'internationalisation du droit : pathologie ou métamorphose de l'ordre juridique ? ». Un ouvrage restituant les travaux du réseau franco-brésilien est en cours de préparation.

\section{Laboratoire de physiologie de la perception et de l'action UMR 7152 CNRS-Collège de France}

\section{Responsable : Sidney WIENER}

Le laboratoire de Physiologie de la perception et de l'action (LPPA) poursuit ses recherches avec des approches multidisciplinaires dans le champ des neurosciences intégratives chez l'homme et des modèles expérimentaux animaux. Nos thèmes de recherche sont centrés sur les bases neurales des fonctions cognitives, en mettant l'accent sur les interactions entre perception et action et bases neurales de la mémoire.

\section{RECHERCHE}

\section{Développement perceptif et intégration inter-hémisphérique}

Équipe : Chantal MILLERET, Jérôme RIBOT, Alexej GrantYN, Emmanuel BUI QuOC, Yonane Aushana, Cynthia LIONS, Sarah CATTAN, Quentin WelnIARZ, Benjamin COHEN-L'HYVER, Chloé CHÊNET

Nos recherches portent sur le rôle du cortex visuel primaire dans l'élaboration de la perception visuelle chez le mammifère, de la naissance à l'âge adulte, après une vision normale ou après un handicap visuel. Les deux études résumées ci-dessous concernent plus particulièrement le mammifère supérieur adulte ayant toujours eu une vision tout à fait normale.

\section{Communication entre les hémisphères : étude du rôle du corps calleux dans la perception du mouvement}

Les neurones du cortex visuel primaire sont tous sélectifs à chacun des attributs de la scène visuelle: ils répondent préférentiellement à une orientation donnée (horizontale, verticale ou oblique), à une direction de mouvement (vers le haut, vers le bas, etc.) d'un stimulus visuel qui se déplace dans leur champ récepteur. Chez le mammifère supérieur, ces neurones codant pour un même attribut sont en outre organisés en colonnes et celles-ci forment des «cartes corticales » que l'on peut visualiser à partir de la surface corticale avec la technique d'imagerie optique. La 
présente étude s'est focalisée sur les «cartes de direction de mouvement». Plus précisément, elle a eu pour objectif d'étudier la spécificité des connexions interhémisphériques calleuses vis-à-vis de ces cartes fonctionnelles. En combinant imagerie optique et marquage neuronal, l'équipe a déjà démontré que les connexions interhémisphériques présentent une forte spécificité fonctionnelle vis-à-vis des domaines d'orientation : les neurones interhémisphériques relient quasi exclusivement des domaines corticaux de même préférence d'orientation. Usant ici de la même approche, nous avons démontré que la spécificité fonctionnelle des connexions calleuses vis-à-vis des domaines de direction est au contraire relativement faible : les connexions calleuses relient soit des domaines identiques ou de directions opposées entre les hémisphères dans des proportions similaires. Ces résultats suggèrent que les connexions calleuses sont impliquées dans la perception du flux optique au niveau du cortex visuel primaire.

\section{Organisation et origine des cartes de fréquences spatiales}

Contrairement à l'orientation et la direction de mouvement, le fait que la fréquence spatiale soit représentée de façon organisée au niveau du cortex visuel chez le mammifère supérieur reste controversé. À cet effet, plusieurs modèles ont été proposés mais aucun consensus n'a été trouvé. Pire encore, des données récentes indiquent que l'organisation décrite par certains en utilisant la technique d'imagerie optique, réputée pourtant comme étant extrêmement fiable, ne serait en fait que le reflet de réponses non spécifiques. Nous avons réexaminé ici cette question importante en utilisant également l'imagerie optique, mais avec une nouvelle méthode d'acquisition permettant de stimuler le cortex visuel primaire avec une très large gamme de fréquences spatiales, par pas de 0,2 octave. Nous démontrons ainsi que des cartes de fréquences spatiales organisées existent effectivement au niveau du cortex visuel primaire, dans V1, V2 et la zone de transition V1/V2 (TZ). Des caractéristiques propres ont même été identifiées dans chacune de ces régions. Par exemple, un gradient de fréquences spatiales plus « abrupt » qu'ailleurs, impliquant à la fois la voie rétino-géniculo-corticale et le corps calleux, a été identifié au niveau de TZ. La modélisation des données nous conduit en outre à la conclusion selon laquelle les cartes de fréquences spatiales observées dans V1, V2 et TZ sont élaborées en impliquant chacune des «canaux » géniculo-corticaux de fréquences spatiales bien spécifiques.

\section{Mémoire spatiale et navigation}

\section{Équipe : Sidney Wiener, Susan Sara, Erika Cerasti, Julien Catanese, HongYing GaO, Virginie OBERTo, Alice FRUCHART}

Notre recherche cible les mécanismes neuronaux de l'intégration des signaux multi sensoriels de l'environnement et de signaux internes (par exemple émotionnels) aussi bien que de l'élaboration, mémorisation et rappel de représentations épisodique. Nos études ont comme but de mieux comprendre les bases neurales des processus cognitifs nécessaires à la survie de l'animal dans son environnement, et leurs relations avec les comportements adaptatifs, et des associations apprises et rappelées. 


\section{Neuromodulation noradrénergique et consolidation mnésique pendant le sommeil consécutif à l'apprentissage}

Nous avons mise en évidence une augmentation de l'activité des neurones noradrénergiques du Locus Coeruleus (LC) pendant le sommeil lent après un apprentissage ou âpres une réactivation de la mémoire par un rappel. De plus, le décharge des neurones du LC sont synchronise avec des ondulations lent du cortex. Étant donné que les ondulations hippocampiques sont 'groupe' par les ondulations lentes du cortex, la libération de la noradrénaline par les neurones du LC faciliterait ou jouerait un rôle permissif dans les modifications de la plasticité dans l'hippocampe et le cortex pendant le sommeil consécutif à l'apprentissage. Nous mettons à l'épreuve cette hypothèse actuellement avec une approche optogénétique en collaboration avec l'équipe du Pr Mu-Ming Poo de l'Institute of Neuroscience à Shanghai.

Participation aux programmes nationaux: ANR Neurobot et ANR EmOpto (SAMENTA)

\section{Approche probabiliste et perception active}

Équipe : Jacques Droulez, Alain Berthoz, Pierre Bessière, Manuel VIDAL, Daniel Bennequin, Guillaume Thibault, Jean-Luc Petit, Colas AuthiÉ, Najate JeBARA, Bérangère THIRIOUX, Laurent DolLÉ, Steve N'GUYEN, Romain DAVID, Alexandre ConINX, Gabriel SyNNAEVE, Pauline HILT

Nous étudions les interactions entre perception et action dans différentes tâches comportementales telle que la perception des objets et du mouvement propre, le rôle du regard et de la stabilisation de la tête dans la locomotion, l'apprentissage et rôle de l'action dans la mémoire spatiale.

\section{Études des fonctions sensorielles}

Les interactions audio-visuelles sont étudiées dans le cadre de la rivalité binoculaire. Nous avons mesuré l'influence de la stimulation auditive sur les oscillations perceptives et l'influence du stimulus visuel supprimé sur la perception auditive, ainsi que la possibilité d'un apprentissage inconscient conditionné par le percept supprimé. Nous avons également développé une nouvelle méthode d'analyse de la géométrie des canaux semi-circulaires basée sur la reconstruction des lignes de flux centrales. Cette méthode a été appliquée à différentes espèces fossiles ou vivantes et ouvre la voie à une comparaison interspécifique morphologique et fonctionnelle précise. La prise en compte des caractéristiques des fonctions visuelles est également utile à l'amélioration des algorithmes de visualisation scientifique de grands ensembles de données.

\section{Études des fonctions mnésiques}

Nous étudions l'impact de l'activité sur différentes composantes de la mémoire épisodique (ME) dans une tâche de navigation nécessitant des processus décisionnels ou moteurs, en simple ou double tâche. Nous avons montré que l'action peut améliorer certaines composantes de la ME mais uniquement lorsque l'action implique un processus simple. Nous étudions également la capacité de mémoriser des 
environnements 3D. Des premières études suggéraient que les sujets mémorisent préférentiellement l'organisation spatiale par plancher. Nous avons mené une étude récente, en collaboration avec $\mathrm{EDF}$, qui montre que cette préférence résulte essentiellement de la façon dont l'environnement a été exploré pendant l'apprentissage.

\title{
Bases neurales des relations à autrui
}

Nous avons décrit différentes phases d'activation cérébrales, associées à des rythmes cérébraux spécifiques entre 80 et $400 \mathrm{~ms}$, sous-tendant l'interaction avec autrui, à partir de données EEG de surface et intracrâniales. Nous avons testé notre paradigme moteur permettant de distinguer l'empathie de la sympathie sur une population de patients schizophrènes.

\section{Regard et locomotion}

Dans le cadre du projet européen RoboSoM, nous enregistrons les mouvements du corps et du regard pendant la locomotion humaine. Nous avons montré que le regard anticipe les mouvements de la tête et du corps et que cette anticipation persiste lorsque les sujets se déplacent dans le noir. Nous avons également enregistré les mouvements du corps et l'orientation du regard lors d'activités locomotrices complexes incluant l'évitement et l'enjambement d'obstacles. Ces données nous permettent de mieux comprendre comment les sujets planifient et contrôlent leur trajectoire en fonction de la tâche locomotrice, et de proposer ainsi des solutions bio-inspirées pour le contrôle des robots humanoïdes.

\section{Modélisation probabiliste}

Depuis plusieurs années, nous avons publiés un certain nombre de modèles bayésiens de la sensori-motricité et du comportement humain ou animal. Nous avons montré récemment comment la perception et l'action pouvaient interagir dans la reconnaissance des lettres manuscrites. Une question ouverte est de comprendre les mécanismes qui permettent au cerveau de coder et de calculer les probabilités. Nous explorons l'idée selon laquelle les réseaux biochimiques de la signalisation cellulaire sont capables d'effectuer ces tâches computationnelles. Des modèles probabilistes sont aussi appliqués à la programmation des robots. Par exemple, nous avons développé un modèle probabiliste permettant à un robot de détecter des perturbations posturales inattendues et de programmer les changements d'appui nécessaires au rétablissement de son équilibre.

Participation aux programmes européens : CLONS et RoboSoM.

Participation aux programmes nationaux: Romeo (FUI), Pace, MANDy (ANR), Score.

\section{Rythmes cérébraux et codage neural de la mémoire}

\author{
Équipe : Gabrielle Girardeau, Anne Cei, Nicolas Maingret, Karim El KanBI, \\ Céline Drieu, Marie Goutierre, Michaël Zugaro
}

De nombreux travaux suggèrent que certaines formes de mémoire sont initialement formées dans l'hippocampe puis progressivement transférées vers le cortex pour un stockage à long terme - un processus nommé consolidation mnésique. Notre équipe 
étudie les bases cérébrales de la mise en place et de la consolidation de la mémoire spatiale. Nous enregistrons l'activité de neurones hippocampiques chez des rats en comportement : ces neurones codent la position de l'animal dans l'environnement et sont appelés cellules de lieu.

On savait depuis longtemps que le sommeil favorisait la consolidation, sans pour autant en connaître les mécanismes neurophysiologiques. Nous avons récemment montré que des oscillations rapides et de courte durée qui se produisent dans l'hippocampe pendant le sommeil à ondes lentes sont indispensables pour le stockage à long terme des traces mnésiques. En effet, ces oscillations s'accompagnent d'activations spontanées de cellules de lieu, qui déchargent les unes après les autres dans le même ordre que pendant l'éveil - comme si le rat refaisait en rêve les mêmes déplacements dans son environnement. Ces réactivations de séquences de neurones constituent donc une exploration virtuelle qui permet le renforcement de la mémoire spatiale. Mais comment ces séquences de neurones se forment-elles initialement pendant l'exploration ?

Pour tester les différentes hypothèses dans la littérature, nous perturbons le fonctionnement normal de l'hippocampe en déplaçant des rats en marche arrière sur un train électrique. De plus, afin de générer dans l'hippocampe les oscillations thêta qui servent normalement d'horloge de référence pour les cellules de lieu lorsque les rats se déplacent activement, le train est doté d'un tapis roulant miniature. Nos résultats préliminaires montrent que l'hippocampe s'adapte parfaitement à ces conditions délibérément artificielles : les séquences de neurones s'inversent et reflètent bien le déplacement en marche arrière. Ceci indique que l'ordre de décharge des cellules de lieu n'est pas contrôlé par la position des repères visuels (qui reste la même dans les deux sens de déplacement) ni par les connexions anatomiques entre cellules de lieu (qui ne changent pas non plus selon le sens de déplacement). En revanche, nos données sont compatibles avec l'hypothèse que les séquences de neurones résultent d'une organisation temporelle intrinsèque fine liée au rythme thêta.

Ces résultats indiquent donc que les séquences de neurones sont générées par l'hippocampe, de manière endogène et non contrôlée par des entrées sensorielles, grâce à un mécanisme temporel. Et comme les cellules de lieu codent l'espace, il s'agit donc in fine d'un mécanisme de génération de séquences spatio-temporelles. Ceci est compatible avec le rôle crucial de l'hippocampe chez l'homme pour la formation de la mémoire épisodique, une forme de mémoire qui associe des événements à leur contexte spatial et temporel.

\section{PUBLICATIONS 2011-2012}

Bastin J., Committeri G., Kahane Ph., Galati G. Minitti L., Lachaux J.-P. et Berthoz A., «Timing of posterior parahippocampal gyrus activity reveals multiple scene processing steps », Human Brain mapping, 2012 [doi: 10.1002/hbm.21515].

Battaglia F.P., Benchenane K., Sirota A., Pennartz C.M. et Wiener S.I., « The hippocampus: hub of brain network communication for memory », Trends Cogn. Sci., 15(7), 2011, 310-18.

Berthoz A. et Thirioux B., «A spatial and perspective change theory of the difference between sympathy and empathy », Paragrana, 19(1), 2011, 32-61.

Boë L.-J., Schwartz J.-L., Granat J., Heim J.-L., Serrurier A., Badin P., Captier G. et Bessière P., «L'émergence de la parole: Aspects historiques et épistémologiques d'une nouvelle réarticulation », Faits de Langues, 37, 2011, 15-67. 
Bui Quoc E. ${ }^{1}$, Ribot J.*, Quenech'du N., Doutremer S., Lebas N., Grantyn A., Aushana Y. et Milleret $\mathrm{C}^{*}$, «Asymmetrical interhemispheric connections develop in cat visual cortex after early unilateral convergent strabismus: Anatomy, physiology and mechanisms », Frontiers in Neuroanatomy, 5, 2011, 1-29.

Catanese J., Cerasti E., Zugaro M., Viggiano A. et Wiener S.I., « Dynamics of decisionrelated activity in hippocampus », Hippocampus, 2012 [doi: 10.1002/hipo.22025].

Cleret de Langavant L., Trinkler I., Remy P., Thirioux B., McIntyre J., Berthoz A., Dupoux E. et Bachoud-Lévi A.C., «Viewing another person's body as a target object: A behavioural and PET study of pointing », Neuropsychologia, 50(8), 2012, 1801-1813.

Cleret de Langavant L., Remy P., Trinkler I., McIntyre J., Dupoux E., Berthoz A. et Bachoud-Lévi A.C., "Behavioral and neural correlates of communication via pointing », PloS One, 6(3), 2011, 1-10.

Chaperon Th., Droulez J. et Thibault G., «Reliable camera pose and calibration from a small set of point and line correspondences: a probabilistic approach », Computer Vision and Image Understanding, 115(5), 2011, 576-575.

Coninx A., Bonneau G.-P., Droulez J. et Thibault G., « Visualization of uncertain scalar data fields using color scales and perceptually adapted noise », Applied Perception in Graphics and Visualization, 2011.

Eschenko O., Magri C., Panzeri S. et Sara S.J., « Noradrenergic neurons of the locus coeruleus are phase locked to cortical up-down states during sleep », Cereb Cortex, 22(2), 2012, 426-35.

Falotico E., Laschi C., Dario P., Bernardin D. et Berthoz A., «Using trunk compensation to model head stabilization during locomotion", Humanoid Robots (11th IEE-RAS Int. Conf.), 2011, 440-445.

Farkhatdinov I., Hayward V. et Berthoz A., « On the benefits of head stabilization with a view to control balance and locomotion in humanoids », Humanoid Robots (11th IEEE-RAS Int. Conf.), 2011, 147-152.

Gais S., Rasch B., Dahmen J.C., Sara S. et Born J., « The memory function of noradrenergic activity in non-REM sleep », J. Cogn. Neurosci., 23, 2011, 2582-2592.

Garcia S., Léna C., Meyrand P., Pouzat C., Shulz D.E. et Zugaro M., « Editorial: Neuronal ensemble recordings in integrative neuroscience », J. Physiol. Paris, 106(3-4), 2012, 57.

Gilet E., Diard J. et Bessière P., «Bayesian Action-Perception Computational Model: Interaction of Production and Recognition of Cursive Letters », PLoS One, 6(6), 2011.

Girardeau G. et Zugaro M., «Hippocampal ripples and memory consolidation », Curr. Opin. Neurobiol., 21(3), 2011, 452-59.

Lambrey S., Doeller Ch., Berthoz A. et Buergess N., «Imaging being somewhere else: Neural basis of changing perspective in space », Cerebral Cortex, 22(1), 2012, 166-174.

Landgraf S., Amado I., Berthoz A., Krebs M.O. et van der Meer E., « Cognitive identity in schizophrenia: Vision, space, and body perception from prodrome to syndrome », Current Psychiatric Reviews, 8(2), 2012, 119-139.

Meilinger T., Berthoz A., Wiener J.M., «The integration of spatial information across different viewpoints », Mem. Cognit., 2011, sous presse.

Menzocchi M., Santarcangelo E.L., Carli G. et Berthoz A., «Hypnotizability-dependent accuracy in the reproduction of haptically explored paths », Exp. Brain. Res., 216(2), 2012, 217-233.

Moulin-Frier C., Laurent R., Bessière P., Schwartz J.-L. et Diard J., « Adverse conditions improve distinguishability of auditory, motor and perceptuo-motor theories of speech

1. Contributeurs égaux. 
perception: an exploratory Bayesian modeling study », Language and Cognitive Processes, 2012, sous presse.

Moulin-Frier C., Schwartz J.-L., Diard J. et Bessière P., «Emergence of articulatoryacoustic systems from deictic interaction games in a "vocalize to Localize" framework", Primate Communication, ad Human Language Vocalisation, gestures, imitation and deixis in humans and non-humans, 2011, 193-220.

N'Guyen S., Droulez J. et Berthoz A., « A probabilistic model of equilibrium perception », Field Robotics, 2011, 839-846.

Olivé I. et Berthoz A., «Combined induction of rubber-hand illusion and out-of-body experiences », Front Psychol., 3, 2012, 128.

Pelissolo A., Zaoui M., Aguayo G., Yao S.N., Roche S., Ecochard R., Gueyffier F., Pull C., Berthoz A., Jouvent R. et Cottraux J., «Virtual reality exposure therapy versus cognitive behavior therapy for panic disorder with agoraphobia: A randomized comparison study », $J$. CyberTherapy \& Rehab., 5(1), 2012, 35-43.

Petrovskaya A., Perrollaz M., Oliveira L., Spinello L., Triebel R., Makris A., Yoder J.-D., Urbano N., Laugier C. et Bessière P., « Awareness of Road Scene Participants for Autonomous Driving », Springer Handbook of Intelligent Vehicles, 2012.

Pham Q.C., Berthoz A., Hicheur H., «Invariance of locomotor trajectories across visual and gait direction conditions », Exp. Brain Res., 210(2), 2011, 207-15.

Poirel N., Vidal M., Pineau A., Lanoë C., Leroux G., Lubin A., Turbelin M.-R., Berthoz A. et Houdé O., «Evidence of different developmental trajectories for length estimation according to egocentric and allocentric viewpoints in children and adults », Exp. Psychol., 58(2), 2011, 142-146.

Senot P., Zago M., Le Séarch A., Zaoui M., Berthoz A., Lacquaniti F. et McIntyre J., «When Up is Down in 0g: How Gravity Sensing Affects the Timing of Interceptive Actions », J. Neurosci., 32(6), 2012, 1969-1973.

Synnaeve G. et Bessière P., «A Bayesian Model for Plan Recognition in RTS Games applied to StarCraft», Proc Seventh Artificial Intelligence and Interactive Digital Entertainment Conf (AIIDE 2011), 2011.

Synnaeve G. et Bessière P., «A Bayesian Model for RTS Units Control applied to StarCraft », Proceedings of IEEE CIG 2011 Computational Intelligence and Games, 2011.

Synnaeve G. et Bessière P., «A Bayesian Model for Opening Prediction in RTS Games with Application to StarCraft », Proceedings of IEEE CIG 2011 Computational Intelligence and Games, 2011.

Tani T.*, Ribot J.*, O’Hashi K. et Tanaka S., « Parallel development of orientation maps and spatial frequency selectivity in cat visual cortex », Eur. J. Neurosci., 35(1), 2012, 44-55.

Vidal J.R., Freyermuth S., Jerbi K., Hamamé C.M., Pssandon T., Bertrand O., Minotti L., Kahane P., Berthoz A. et Lachaux J.P., «Long-distance amplitude correlations in the high gamma band reveal segregation and integration within the reading network », J. Neurosci., 32(19), 2012, 6421-6434.

Wiener J.M., Berthoz A. et Wolbers T., «Dissociable cognitive mechanisms underlying human path integration », Exp. Brain Res., 208(1), 2011, 61-71.

Zanelli G., Cappa P., Petrarca M. et Berthoz A., « Vestibular and proprioceptive estimation of imposed rotation and spatial updating in standing subjects », Gait Posture, 33(4), 2011, 582-587. 